\title{
Regional distribution of atrial electrical changes induced by stimulation of extracardiac and intracardiac neural elements
}

\begin{abstract}
Autonomic nerves and intrinsic cardiac neural elements are known to influence the electrophysiologic and dynamic properties of the heart. This study describes the regional distribution in the canine atria of electrophysiologic effects induced by stimulation of the right and left cervical vagosympathetic complexes, the right atrial ganglionated plexus, and the right and left stellate ganglia. Local atrial effects were determined from changes in QRST area of unipolar electrograms recorded from multiple sites with plaque electrodes sewn onto the atria in 16 anesthetized dogs. Results: (1) Although being very consistent in any given preparation, atrial changes varied between animals when similar neural structures were stimulated. (2) Among the common features identified between preparations, consistent effects were induced by neural stimulation in the region of the sinus node, indicating that this atrial region is the most richly innervated. (3) All other regions of the atria could be affected by stimulation of either right-sided or left-sided efferent nerves. (4) Responses to right atrial ganglionated plexus stimulation after atropine administration indicated that the corresponding fat pad contains both sympathetic and parasympathetic neural elements. Conclusion: This study demonstrates that there is considerable overlapping of atrial innervation affecting all regions of the atria, as well as the sinus node region. (J THORAC CARDIOvASC SURG 1995;109:377-88)
\end{abstract}

Pierre L. Pagé, $\mathrm{MD},{ }^{\mathrm{a}, \mathrm{b}}$ Nazih Dandan, $\mathrm{MD},{ }^{\mathrm{a}, \mathrm{b}}$ Pierre Savard, $\mathrm{PhD},{ }^{\mathrm{a}, \mathrm{c}}$

Réginald Nadeau, $\mathrm{MD},{ }^{\mathrm{a}} \mathrm{J}$. Andrew Armour, $\mathrm{MD}, \mathrm{PhD},{ }^{\mathrm{d}}$ and René Cardinal, $\mathrm{PhD},{ }^{\mathrm{a}}$

Montreal, Quebec, and Halifax, Nova Scotia, Canada

$\mathrm{E}$ lectrical stimulation of intrinsic cardiac neural elements has been demonstrated to influence electrophysiologic and dynamic properties of the human heart. ${ }^{1,2}$ It has also been proposed as a method to

From the Centre de Recherche de l'Hôpital du Sacré-Coeur de Montréal, ${ }^{\text {a }}$ the Department of Surgery, ${ }^{\mathbf{b}}$ Université de Montréal, and the Ecole Polytechnique de Montréal, ${ }^{\mathrm{c}}$ Montreal, Quebec, Canada, and the Department of Physiology and Biophysics, ${ }^{\mathrm{d}}$ Dalhousie University, Halifax, Nova Scotia, Canada.

Supported by grants from the Heart and Stroke Foundation of Québec, the Medical Research Council of Canada, and a donation from Medtronic Inc.

Received for publication Jan. 28, 1994.

Accepted for publication July 12, 1994.

Address for reprints: Pierre L. Pagé, MD, Centre de recherche, Hôpital du Sacré-Coeur de Montréal, 5400 Gouin Blvd. West, Montreal, Quebec, Canada H4J 1 C5.

Copyright (c) 1995 by Mosby-Year Book, Inc.

0022-5223/95 $\$ 3.00+0 \quad \mathbf{1 2 / 1 / 5 9 1 5 5}$ modify supraventricular arrhythmias. ${ }^{3,4}$ Moreover, any autonomic imbalance occurring after cardiopulmonary bypass procedures may contribute, at least in part, to the pathophysiology of postoperative atrial arrhythmias., 5

Autonomic nerves affect cardiac chronotropism, dromotropism, and inotropism through their efferent projections to the sinus node, atrioventricular (AV) node, and atrial musculature. Thus, methods used to study the functional innervation of the atria in the canine heart, the preferred preparation in most investigations of cardiac innervation, have relied on changes in heart rate and pacemaker location, AV conduction time, and contractile responses. ${ }^{6-9}$ Parasympathetic postganglionic efferent neurons have been localized in the right atrial ganglionated plexus, a collection of neural elements contained in a triangular fat pad on the ventral aspect of the right atrial free wall (pulmonary vein fat pad), as well as a ganglionated plexus located in 
fatty tissues overlying the junction of the inferior vena cava and inferior left atrium. ${ }^{10-13}$ Their functional properties and, particularly, the hypothesis that selective parasympathetic efferent innervation of the sinus node and AV node may occur through the right atrial and inferior left atrial ganglionated plexi, respectively, were investigated through selective stimulation ${ }^{10,14}$ or surgical removal. ${ }^{15-18}$ However, little information is to be found on functional pathways to regions of the atria other than the sinus and AV nodes. Furthermore, previously reported data indicating that the right atrial ganglionated plexus might contain sympathetic as well as parasympathetic efferent neural elements ${ }^{14}$ need to be further investigated.

The repolarization phase of the cardiac action potential, too, is very sensitive to parasympathetic and sympathetic nerve stimulation. Therefore, refractory period determinations have been used to study regional atrial effects in response to stimulation of the right and left vagosympathetic complexes and stellate ganglia. ${ }^{19-21}$ However, the use of refractory period measurements is limited by the fact that only one site can be sampled at a time and that each determination requires several seconds to complete. Several variables that can be extracted from the unipolar electrogram-QRST area, ${ }^{22}$ activationrecovery interval, ${ }^{23}$ QT interval, and T-wave amplitude $^{24}$ - have been used to determine ventricular patterns of efferent sympathetic innervation (for review, see reference 25). When used in conjunction with a multichannel recording system and multiple electrode array, variables extracted from unipolar electrograms can be measured from many sites on a beat-to-beat basis, so that the spatial distribution of electrical responses induced by stimulation of specific efferent autonomic neural elements is analyzed with a high degree of spatial and temporal resolution.

The area of QRST deflections in the unipolar waveform is a measure of electrical forces generated by local variations in action potential duration. ${ }^{26}$ This variable is measured by an integration process in which the areas of positive deflections are added and the areas of negative deflections are subtracted, positive and negative deflections being defined with reference to the isoelectric diastolic baseline. This variable is theoretically independent of the sequence of activation. Previous reports have suggested that QRST deflection area may be useful to determine changes in recovery properties. ${ }^{22,27} \mathrm{Re}-$ cently, we $\mathrm{e}^{28}$ have reported studies in which QRST area changes at multiple sites were used to map ventricular patterns of efferent sympathetic innervation. In our experience, this method avoids problems encountered during determination of QT intervals at individual recording sites because of ambiguous end points, that is, multiphasic or lowamplitude $T$ waves and slurred transition back to isoelectric baseline. In the present study, we used QRST area measurements to detect regional changes in atrial electrical activity induced by stimulation of individual neural elements, namely, the right and left vagosympathetic complexes, the right and left stellate ganglia, and the right atrial ganglionated plexus. This study provides a descriptive analysis of the specific projections of efferent autonomic neural elements onto the atria.

\section{Methods}

Sixteen mongrel dogs, weighing 25 to $30 \mathrm{~kg}$, were anesthetized with sodium thiopental $(15 \mathrm{mg} / \mathrm{kg})$ and $\alpha$-chloralose $(100 \mathrm{mg} / \mathrm{kg})$. Anesthesia was maintained with $\alpha$-chloralose administration, as required. After initiation of positive-pressure ventilation with a Harvard respirator (Harvard Apparatus Co., Inc., S. Natick, Mass.), the right and left cervical vagosympathetic trunks were exposed via a midline neck incision and sectioned. A bilateral thoracotomy was performed in the fifth intercostal space. Loose ligatures were placed around the right and left stellate ganglia. The heart was placed in a pericardial cradle. Bipolar electrodes used for pacing were sutured onto the left atrial appendage and right ventricle. Atrial and ventricular electrograms, a lead II electrocardiogram, and systemic arterial pressure were monitored on a polygraph (Nihon Kohden Corp., Tokyo, Japan) and stored on magnetic tape (Hewlett-Packard instrumentation recorder, Hewlett-Packard Co., Palo Alto, Calif.) throughout the experiments.

To facilitate the analysis of several atrial unipolar electrograms without interference from ventricular QRS complexes, complete AV block was induced by formaldehyde injection ( 0.1 to $0.5 \mathrm{ml}$ of a $37 \%$ solution) into the His bundle region with a 22-gauge needle introduced into the right atrial cavity through the right atrial free wall. The needle was guided by palpating the triangle of Koch with the index finger. Between periods of nerve stimulation, the ventricles were paced at a frequency of 100 beats $/ \mathrm{min}$.

Atrial mapping. Unipolar electrograms were simultaneously recorded from multiple sites equally distributed over the total epicardial surface of the right and left atria by means of flexible templates made from a double layer of reinforced Silastic silicone rubber sheets (Dow Corning Corp., Midland, Mich.) carrying 63 (five preparations) or 127 (eleven preparations) recording contacts (Fig. 1). Five such templates were shaped to be implanted over the right atrial free wall, posteroinferior wall of the left atrium and coronary sinus, posterior aspect of the left atrium (template inserted between the inferior pulmonary veins), left atrial free wall, and interatrial band, respectively. Unipo- 
lar electrograms recorded with reference to the Wilson central terminal were amplified, filtered $(0.05$ to $200 \mathrm{~Hz})$, multiplexed, digitized at $1 \mathrm{KHz}$, and stored on a hard disk with a 128-channel data acquisition system (Institut de Génie Biomédical, Université de Montréal). ${ }^{29}$ Files were later retrieved for data analysis, as described herein.

Experimental protocol. Selected nerves were stimulated supramaximally with bipolar stainless steel electrodes connected to a Grass SD9 square-wave stimulator $(20 \mathrm{~Hz}, 2 \mathrm{msec}, 5$ to $10 \mathrm{~V}$ ) (Grass Instrument Co., Quincy, Mass.). The right and left cervical vagosympathetic complexes, the right and left stellate ganglia, and three to four loci in the right atrial ganglionated plexus were stimulated, in turn, for a period of 25 seconds during which ventricular pacing was interrupted (Fig. 2). Responses to neural stimulation were studied during spontaneous rhythm and constant atrial pacing at a cycle length of 350 msec to avoid possible rate-dependent changes in repolarization. Digitized electrograms were recorded with the computer system throughout the period of stimulation and stored on disk as 26-second files of continuously recorded data. Data presented below were obtained at the time of maximal chronotropic changes. Data were also stored under baseline conditions between periods of stimulation. Data obtained during each period of neural stimulation were compared with control data obtained before stimulation. To study the effects of sympathetic neural elements possibly existing in the right atrial ganglionated plexus, we repeated the stimulation of this structure under muscarinic blockade (atropine sulfate $1 \mathrm{mg} / \mathrm{kg}$ given intravenously).

Data analysis. QRST isoarea maps were generated to determine the magnitude and regional distribution of repolarization changes in response to stimulation of individual nerves. With the use of custom-made computer software, files containing 1 second of data were retrieved from disk and each signal was displayed on a video screen to adjust a baseline joining successive isoelectric segments occurring between the T and QRS waves of atrial electrograms. The net area under the QRS complex and $\mathrm{T}$ wave of each electrogram was computed by an integration process by means of a modification of Simpson's technique and a custom software that added the values of sample sectors multiplied by the duration of each sampling period (Institut de Génie Biomédical, Université de Montréal). ${ }^{22,25,27,28}$ For overall quantitative assessment of repolarization changes, the difference between pre-stimulation QRST area value and that measured during stimulation was calculated at each site (Fig. 3). The statistical distributions of changes induced in response to stimulation of each nerve were plotted by means of the SYSTAT software package. ${ }^{30}$ One-way analysis of variance (ANOVA) was performed with the use of data from electrode sites for which repeated measurements were available for all nerves. A significant outcome of the ANOVA was followed by univariate $F$ test comparisons between responses to each nerve, on one hand, and changes between two sets of data obtained under basal conditions (control trial), on the other hand (the Bonferroni correction for multiple comparisons with the same control was used). So that regional effects could be investigated, the atrial grid was conceptually divided into eight
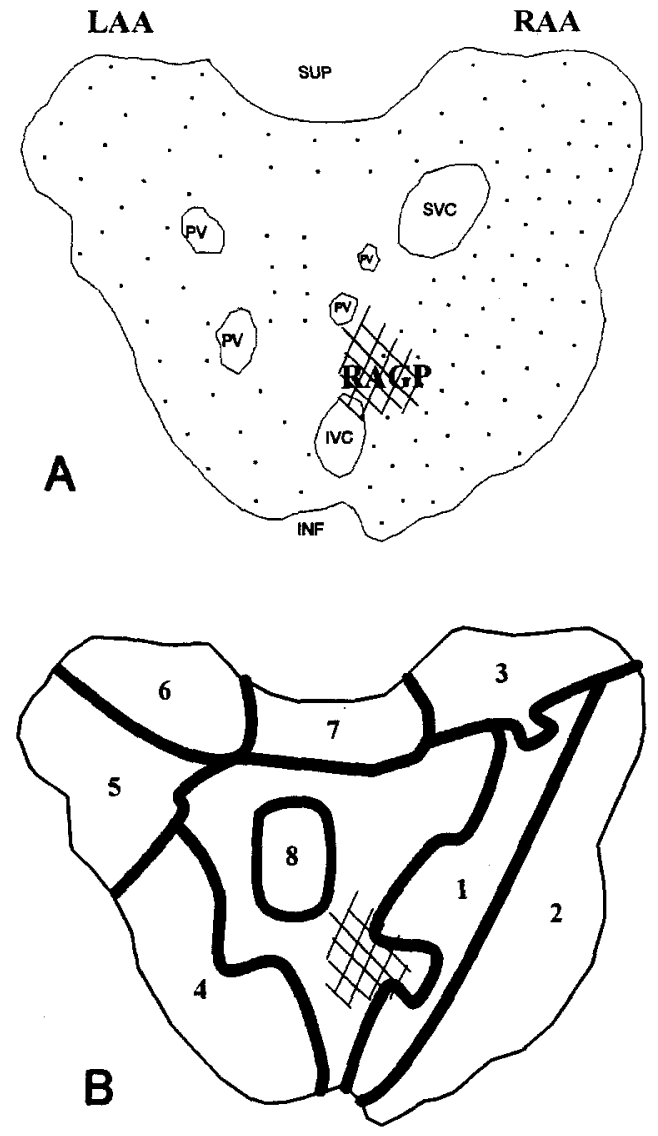

Fig. 1. Mapping grid (A) and subdivision of the atrial surface into eight anatomic regions $(\mathbf{B})$. The atrial surfaces are unfolded and viewed from a posterior projection. Anatomic orifices are indicated by abbreviations. A, Dots indicate sites where a total of 127 unipolar electrodes were recorded. SUP, Superior aspect; $I N F$, inferior aspect; $I V C$, orifice of the inferior vena cava; $L A A$, left atrial appendage; $P V$, pulmonary veins; $R A A$, right atrial appendage; $R A G P$, right atrial ganglionated plexus (hatched area). B, Region 1, sinus node and medial right atrial wall; region 2, right atrial free wall; region 3 , right atrial appendage; region 4 , inferior left atrial wall; region 5 , superior left atrial free wall; region 6 , medial left atrial appendage; region 7 , interatrial band; region 8 , midposterior left atrium.

anatomic regions (as indicated in Fig. 1, B) and statistics for each region were computed from algebraic summation (see below, Fig. 4) or absolute values, including data from all experiments. The significance of changes computed for each region in response to stimulation of each nerve was tested with respect to the null hypothesis (no change) with the $t$ test. Statistics are presented as mean \pm standard deviation. Atrial rates were compared between basal state and each period of neural stimulation by means of the $t$ test. Changes in activation patterns were studied from isochronal maps generated by methods previously de- 

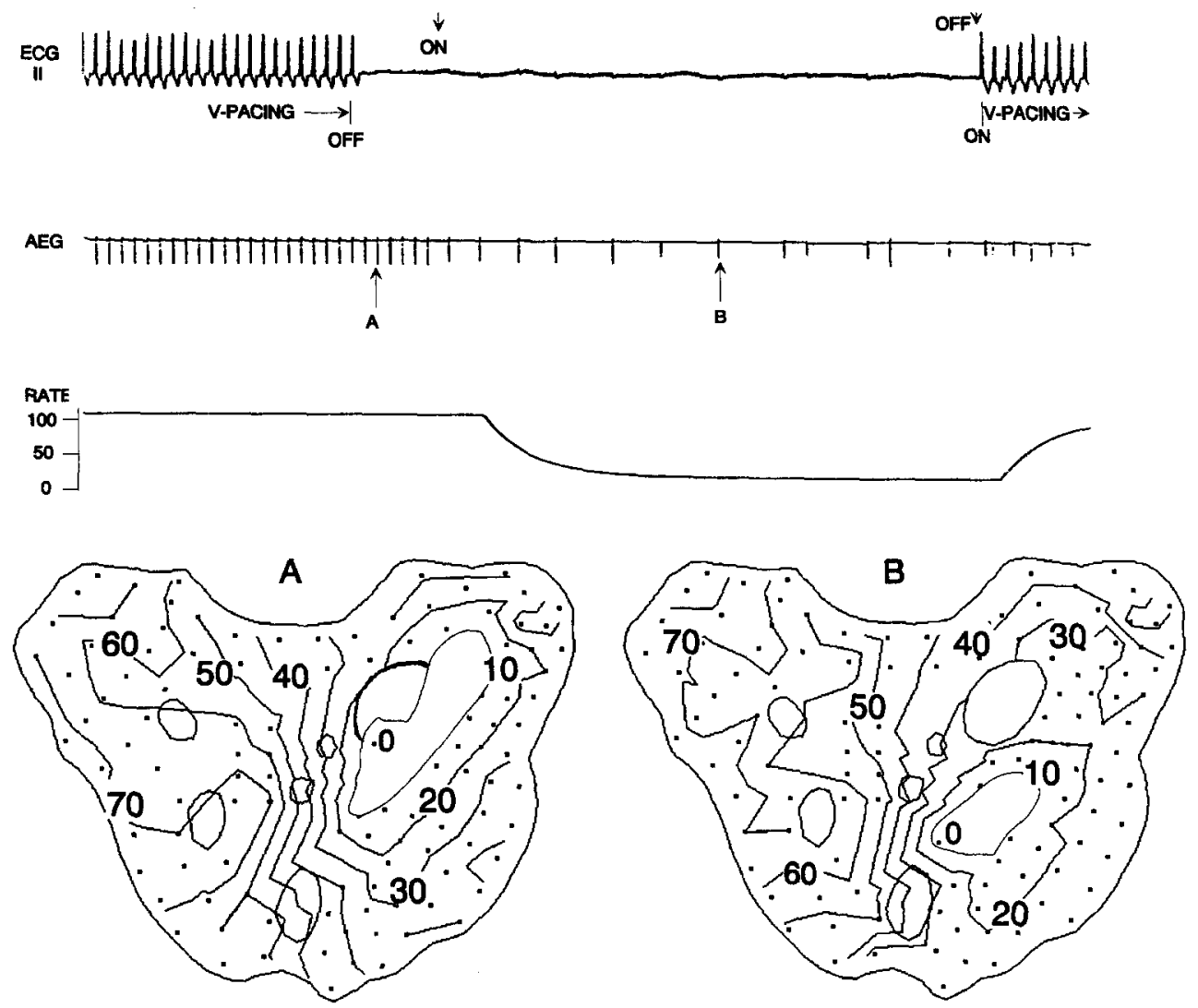

Fig. 2. Pacemaker shift in response to stimulation of the right vagosympathetic complex (RVSC). The upper tracings (lead II electrocardiogram [ECG] and atrial electrogram [AEG] show that, in canine preparations with AV dissociation, ventricular pacing (V-pacing) was temporarily interrupted to study atrial activity without interference from ventricular activity. The atrial tachogram (third trace) shows a decrease in spontaneous rate on RVSC stimulation. Isochronal maps are shown for a sinus beat (A) and atrial beat (B) occurring during RVSC stimulation. In A, the earliest activation (0 to 10 isochrone line) was detected in the region of the sinus node. Note that the region of earliest activity was shifted inferiorly in the right atrial wall during RVSC stimulation. Isochronal lines are drawn at $10 \mathrm{msec}$ intervals.

scribed. ${ }^{4}$ The incidence, among preparations, of specific regional effects was assessed by counting the number of preparations that displayed QRST area changes beyond a threshold of $\pm 100 \mathrm{mV} \cdot \mathrm{msec}$ corresponding to 2 standard deviations of changes obtained when measurements were repeated under basal conditions. The spatial distribution of changes in response to stimulation of a given nerve was represented as an isoarea map generated by plotting QRST area values (millivolts times milliseconds) recorded at each recording site on the atrial grid using color-coded zones including points displaying values within a given range (see below, Fig. 5). The epicardial area (square centimeters) encompassed by neurally induced changes was calculated on the basis of the number of electrode sites displaying changes beyond the $\pm 100 \mathrm{mV} \cdot \mathrm{msec}$ threshold, assuming a mean area of $0.6 \mathrm{~cm}^{2}$ covered by each recording site. Experimental procedures were carried out in accordance with the guidelines of the Canadian Council for Animal Care ${ }^{31}$ and closely monitored by an institutional animal care committee.

\section{Results}

Effects on atrial rhythm. Atrial rate decreased, in all preparations, in response to stimulation of either the right or left cervical vagosympathetic complexes (from $116 \pm 29$ beats $/ \mathrm{min}$ to $48 \pm 60$ beats/min, $p<$ 0.001 ), and there was a concomitant caudal shift of the atrial pacemaker (see Fig. 2). Stimulation of the right stellate ganglion increased the atrial rate in all preparations ( $171 \pm 48$ beats $/ \mathrm{min}, p<0.001$ ), but stimulation of the left stellate ganglion had this effect in only 11 of 16 animals ( $141 \pm 34$ beats $/ \mathrm{min}$, $p<0.01$ ). Significant bradycardia (at least $20 \%$ increase in control cycle length) occurred in response to electrical stimulation of the right atrial ganglionated plexus at a mean of three loci per preparation. Atrial fibrillation was initiated when the stimulating electrode was positioned in a periph- 


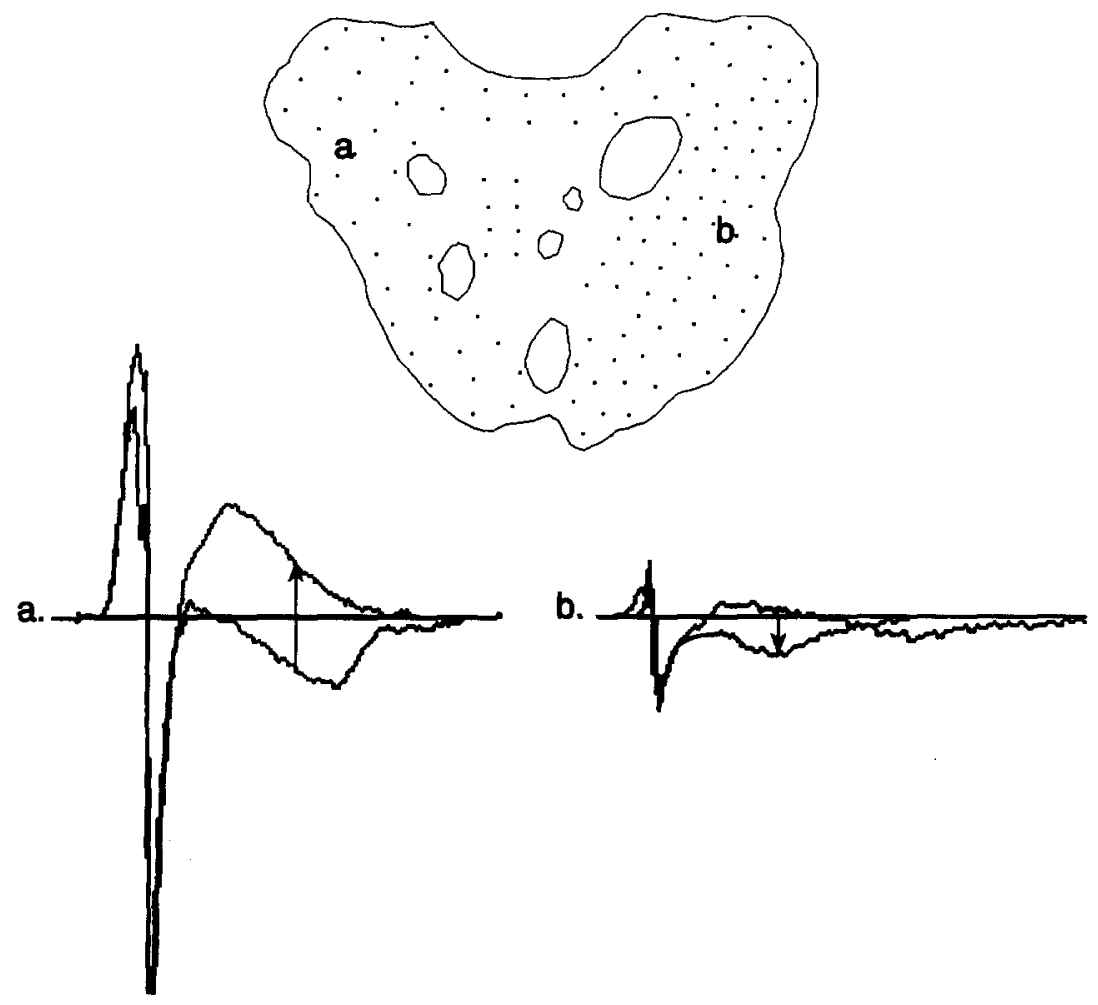

Fig. 3. QRST area changes during neural stimulation. Traces show superimposed unipolar electrograms recorded under basal conditions and during stimulation of the LVSC. Two selected electrograms showing changes of opposite polarity are illustrated. Recording sites are indicated on the atrial grid. Neural stimulation affected primarily the $T$ wave (arrows) of unipolar electrograms. These neurally induced changes in $\mathrm{T}$-wave morphology were expressed as positive (left-hand electrogram) or negative (right-hand electrogram) QRST area changes.

eral region of the fat pad or when it penetrated too deeply. Atrial fibrillation also occurred in $20 \%$ of vagosympathetic complex stimulation trials.

Neurally induced QRST area changes. Unipolar atrial electrograms (see Fig. 3) displayed depolarization (QRS) complexes and repolarization (T) waves commencing immediately after the depolarization complex, a classic feature of the atrial electrogram relating to the fact that phases 2 and 3 of atrial action potentials cannot be distinguished. ${ }^{32}$ Under basal conditions, most unipolar electrograms recorded from the atrial surface displayed lowamplitude $T$ waves of either positive or negative polarity. QRST area changes, expressed as differences between values measured during neural stimulation minus those measured before stimulation, consisted primarily of changes in T-wave amplitude and polarity (see Fig. 3). The quality of electrograms allowed calculation of QRST area in $88.4 \%$ of the recordings made under basal conditions and in $84 \%$ to $89 \%$ during neural stimulation.
When measurements were repeated under basal conditions to assess the control variability of QRST area changes, the changes were found to be normally distributed, displaying a mean value close to zero $(-1$ $\mathrm{mV} \cdot \mathrm{msec})$ and a standard deviation of \pm 51 $\mathrm{mV} \cdot \mathrm{msec}$. When each nerve was stimulated, positive and negative changes occurred, but there was a significant overall effect associated with nerve stimulations by comparison with the control situation (ANOVA, $p<0.05$ ). The absolute value of changes (i.e., their magnitude, whether positive or negative) in each atrial region during stimulation of each nerve varied from $56 \pm 47$ to $271 \pm 257 \mathrm{mV} \cdot \mathrm{msec}$. Responses were significant in all regions for all nerves, except for changes in region 8 in response to left stellate ganglion stimulation $(56 \pm 47 \mathrm{mV} \cdot \mathrm{msec})$. When the polarity of changes was taken into consideration, significant changes displaying a dominant polarity (either positive or negative) occurred in specific atrial regions in response to each nerve (see Fig. 4). The most consistent effect was a positive polarity change in the right 

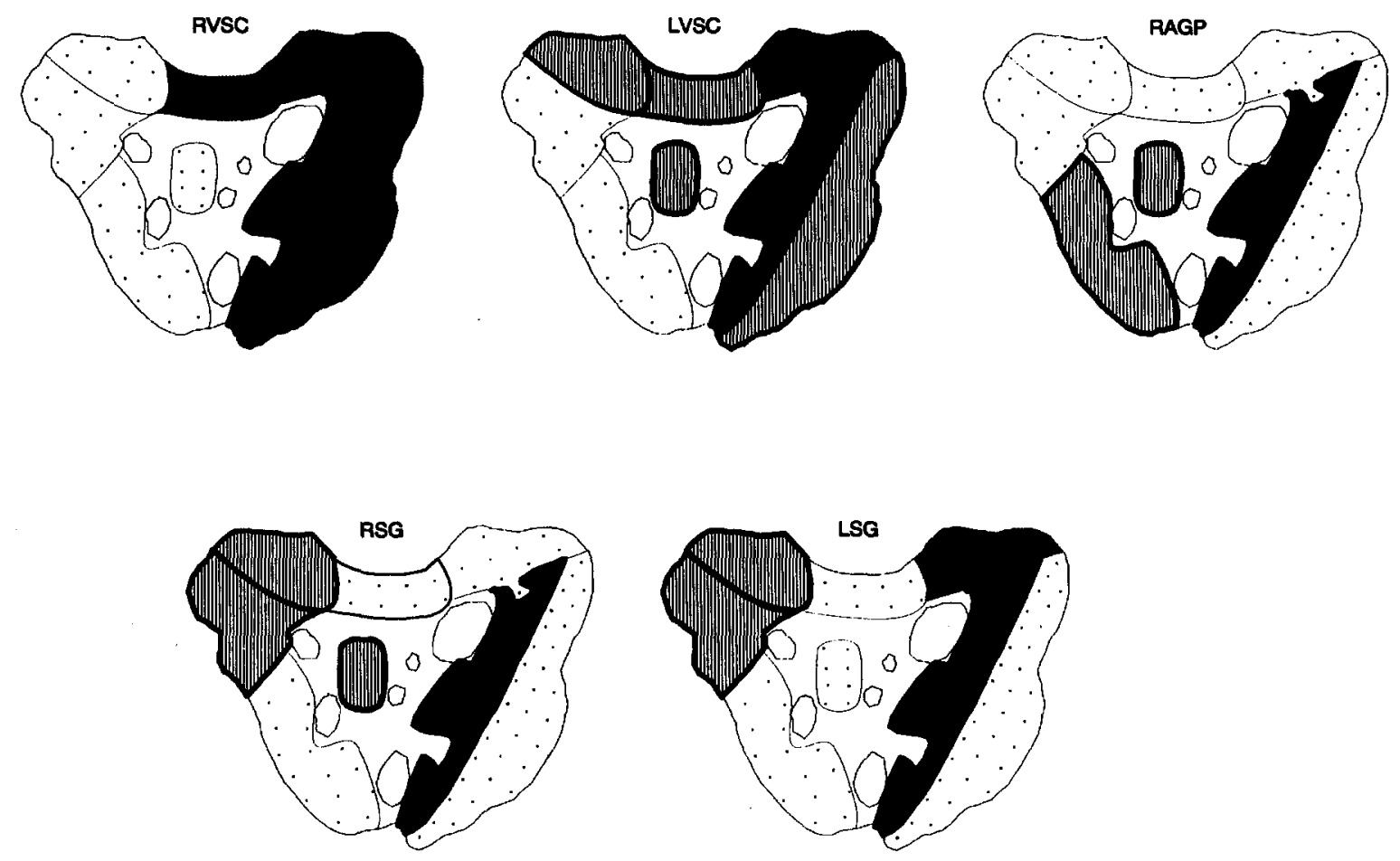

Fig. 4. Regional patterns of QRST area changes in response to stimulation of individual nerves. On each diagrammatic representation of the atrial surface, shaded areas indicate significant changes of a positive (dark) or negative (light) polarity. In response to RVSC stimulation, significant positive changes occurred in the medial right atrial wall (Fig. 1, region 1), the lateral right atrial free wall (region 2), and the interatrial band (region 7). In region 1, changes of a similar polarity but smaller magnitude occurred in during left vagal stimulation (LVSC). In contrast to RVSC stimulation, LVSC produced changes of the opposite polarity in the lateral right atrial free wall and interatrial band (regions 2 and 7). Negative changes also occurred in the superior and mid-posterior left atrium (regions 6 and 8). Stimulation of the neural elements contained in the right atrial fat pad (RAGP) affected mainly the sinus node region but also exerted effects of the opposite polarity on discrete portions of the left atrium. Stimulation of the right and left stellate ganglia induced significant responses in the medial right atrial wall (positive) and left atrial appendage (negative). RVSC, Right vagosympathetic complex; $L V C S$, left vagosympathetic complex; $R A G P$, right atrial ganglionated plexus (fat pad); $R S G$, right stellate ganglion; $L S G$, left stellate ganglion.

Fig. 5. A through D, Isoarea maps of spontaneous rhythms under basal conditions and neural stimulation in selected preparations. Isoarea lines delimit color-coded zones including points where QRST area measurements were within a given $60 \mathrm{mV} \cdot \mathrm{msec}$ range of values (see color coded scale at the bottom). Each isoarea map was photographed from the computer video screen. Increases in T-wave amplitude are shown as positive values (green to blue), whereas reductions of $\mathrm{T}$-wave amplitude or inversion are represented as negative values (orange to brown). The major effects common to the right and left vagosympathetic stimulation and right atrial ganglionated plexus consisted of a green-blue shift in the right atrial free wall. Effects of stellate ganglion stimulation were more variable. Typically, right stellate ganglion stimulation (B and D, RSG maps) induced positive changes in the right atrial wall (eight preparations) and left atrial appendage (seven preparations). In preparation $\mathrm{B}$, left stellate ganglion stimulation (LSG map) induced changes that were restricted to the sinus node region. The two maps obtained after atropine administration (RAGP-AT) show disappearance of positive changes in the right atrium when compared with RAGP maps. RVSC, Right vagosympathetic complex; $L V C S$, left vagosympathetic complex; $R A G P$, right atrial ganglionated plexus (fat pad); $R S G$, right stellate ganglion; $L S G$, left stellate ganglion. 

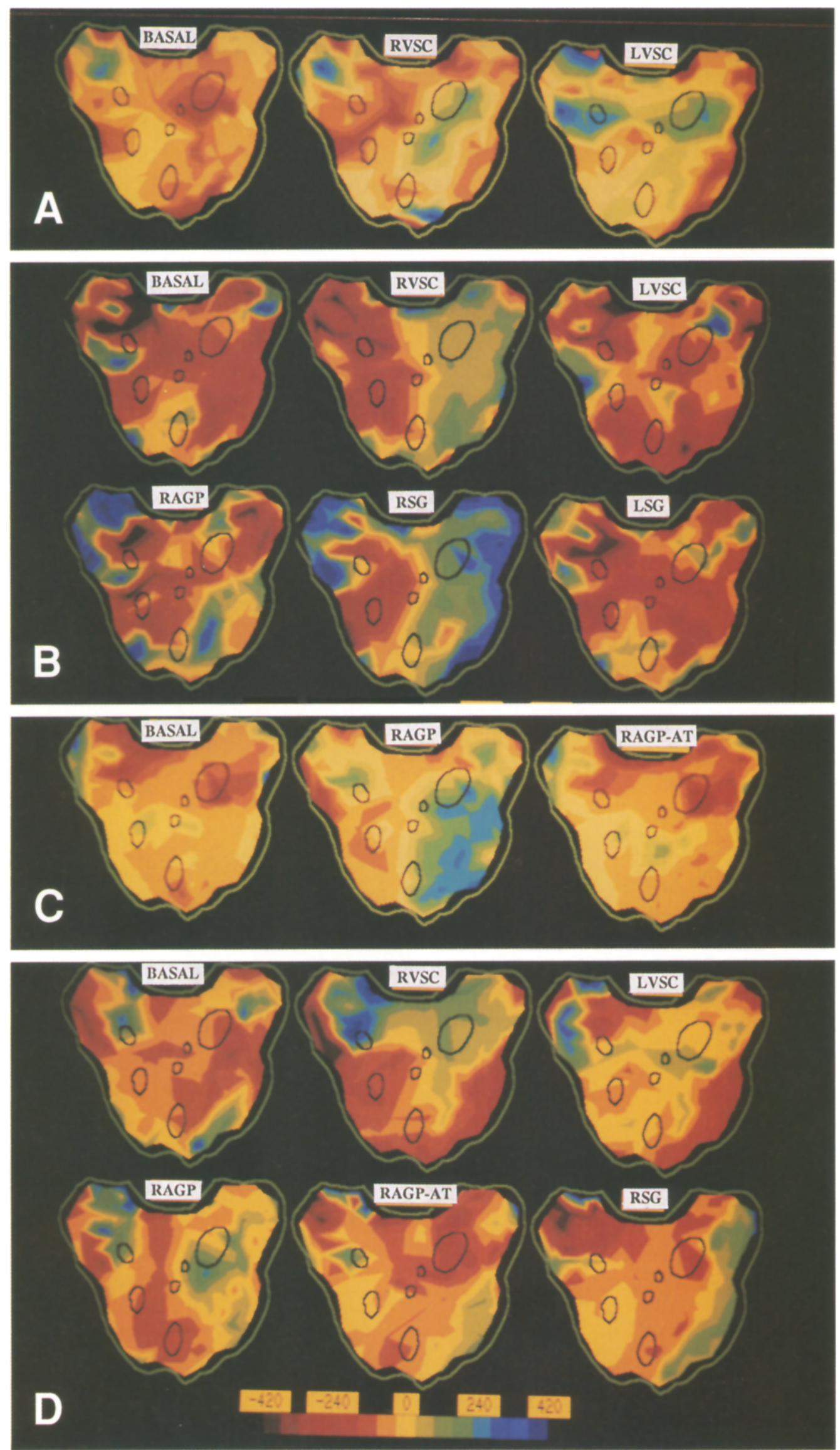

Fig. 5. For legend see opposite page. 
atrial medial free wall (region 1, which includes the sinus node), which occurred in response to stimulation of each nerve. Significant negative changes occurred in the left atrium in response to stimulation of the left vagosympathetic complex, right atrial ganglionated plexus, and right or left stellate ganglia. The results did not differ whether QRST area was calculated during left atrial pacing or spontaneous rhythms.

Neurally induced QRST isoarea map changes. Despite the significant variability occurring between preparations, maps obtained under basal conditions showed typical patterns of QRST area values. In one pattern (six preparations), null or slightly negative QRST area values were identified in the right atrium (Fig. 5, $A$ and $C, B A S A L$ maps). In another pattern (10 preparations), a narrow band of positivity appeared in the lower right atrial free wall (Fig. $5, B$ and $D, B A S A L$ maps). During stimulation of neural structures, the maps generated from spontaneous rhythms were similar to those obtained during left atrial pacing.

Stimulation of the right vagosympathetic complex induced a shift to positive QRST polarity in the right atrial free wall (Fig. 5, $A, B$, and $D, R V S C$ maps). Interanimal variability of responses occurred, as illustrated by the fact that right vagosympathetic complex stimulation induced similar global patterns of effects, albeit with a variable distribution in the right atrium (e.g., Fig. 5, $A$ and $B, R V S C$ maps). Stimulation of the left vagosympathetic complex also induced changes in both atria. The area over which such changes occurred was smaller than those induced by right vagosympathetic complex stimulation $\left(31 \pm 17 \mathrm{~cm}^{2}\right.$ and $43 \pm 10 \mathrm{~cm}^{2}$, respectively, $p<$ $0.01)$. Positive QRST area changes were induced in eight preparations. Typical examples are shown in Fig. 5, $A$ and $D$ (LVSC maps).

When changes occurred in response to stellate ganglion stimulation, they generally occurred in both the right atrial wall and left atrial appendage, but they were restricted to a mean of two atrial regions (Fig. 5, RSG and $L S G$ maps). The changes so induced encompassed $18 \pm 13 \mathrm{~cm}^{2}$ and $14 \pm 15 \mathrm{~cm}^{2}$ for the right and left stellate ganglia, respectively. In a preparation in which left stellate ganglion stimulation did not affect sinus rate, negative changes were found only in region 5 (left atrial appendage), without an effect in the sinus node region.

Stimulation of loci in the right atrial ganglionated plexus produced positive changes in the sinus node area and the right atrial free wall. These effects were similar to those induced by right vagosympathetic complex stimulation but, in 14 preparations, encompassed smaller regions of the right atrium $(31 \pm$ $13 \mathrm{~cm}^{2}$ and $43 \pm 10 \mathrm{~cm}^{2}$, respectively, $p<0.01$; Fig. $5, B, C$, and $D, R A G P$ maps). In one animal, stimulation of a specific locus in the right atrial ganglionated plexus produced an increase in heart rate, suggesting a sympathetic efferent neuronal effect. Accordingly, the isoarea map (not shown) did not exhibit the modifications usually induced by parasympathetic stimulation in the right atrium, showing changes similar to those induced by right stellate ganglion stimulation. In another experiment, positive changes were induced in the left atrial appendage that were not seen when the right vagosympathetic complex was stimulated (Fig. 5, $B$, $R A G P$ and RVSC maps) but that were similar to those generated when the right stellate ganglion was stimulated (Fig. 5, B, RAGP and RSG maps). Efferent sympathetic neural elements in the right atrial ganglionated plexus were also identified after atropine administration in 11 preparations because the induction of positive QRST area changes in the right atrium was inhibited (Fig. 5, $C$ and $D$, $R A G P-A T$ maps), whereas changes similar to those generated in response to right (five preparations) or left (one preparation) stellate ganglion stimulation were induced. In Fig. 5, $D$, an RAGP-AT map is shown next to an RSG map for comparison; similarities between the two interventions can be seen in the right atrium and in the left atrial appendage. These data indicate that the right atrial ganglionated plexus may contain neural elements originating from neural structures other than the right vagosympathetic complex, possibly from the right stellate ganglion.

Incidence of QRST area changes. Despite the fact that similar patterns of QRST area map changes were associated with specific neural structures, stimulation of a given neural structure did not induce changes greater than the $\pm 100 \mathrm{mV} \cdot \mathrm{msec}$ threshold in all atrial regions of all preparations (Fig. 6). The right and left vagosympathetic complexes and the right atrial ganglionated plexus induced changes in all animals (right atrium: 16, 14, and 16 preparations, respectively; left atrium: 14, 14 , and 13 preparations, respectively). In contrast, right and left stellate ganglion stimulation induced changes in the right atrium in nine and six preparations, respectively, and in the left in seven preparations each, but failed to elicit atrial changes in any region in three and four preparations, respectively. 


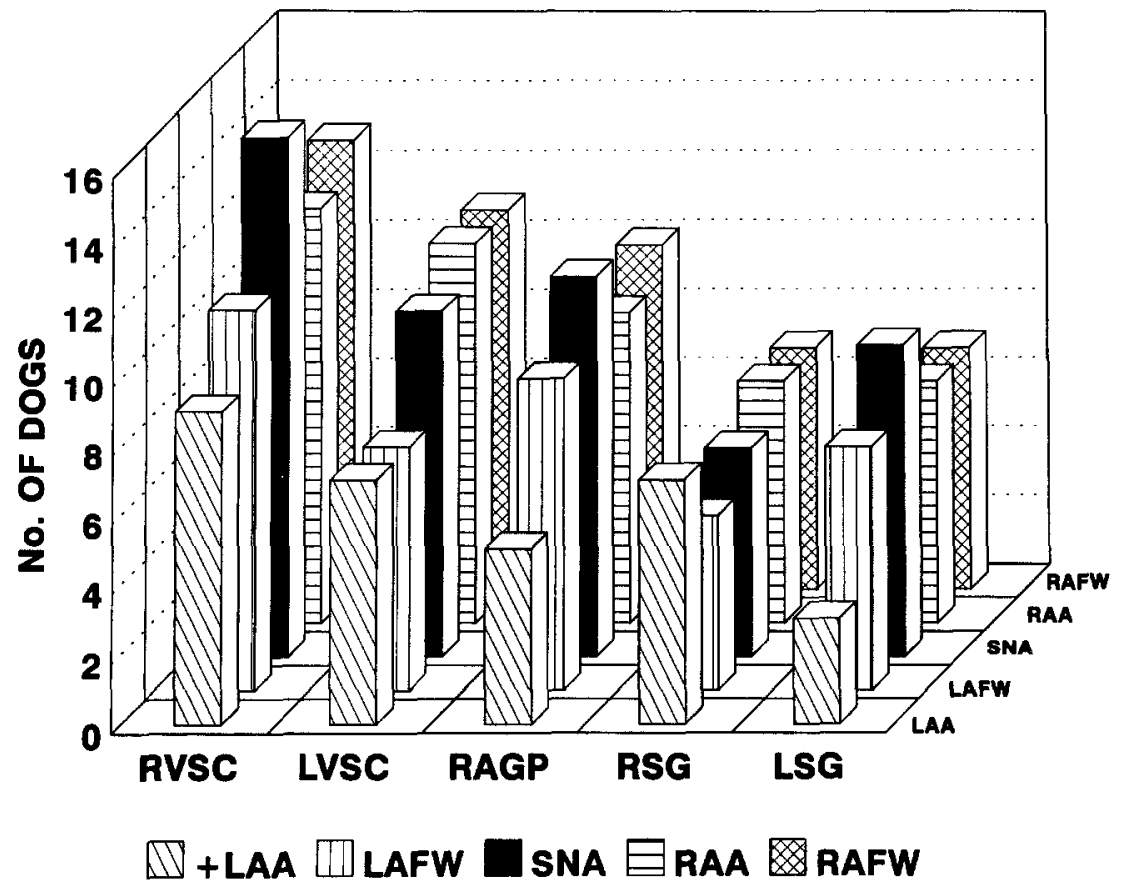

Fig. 6. Histogram representing incidence, among 16 preparations, of QRST area changes beyond \pm 100 $\mathrm{mV} \cdot \mathrm{msec}$ in response to nerve stimulation. The incidence of specific regional effects was assessed by counting the number of preparations that displayed QRST area changes beyond a threshold of \pm 100 $\mathrm{mV} \cdot \mathrm{msec}$ corresponding to 2 standard deviations of repeated measurements made under basal conditions. Abbreviations for nerves are as in Fig. 4. $L A A$, Left atrial appendage (regions 5 and 6 ); $L A F W$, left atrial free wall (regions 4 and 8 ); $R A A$, right atrial appendage (region 3); $R A F W$, right atrial free wall (region 2); $S N A$, sinus node area (region 1 ).

\section{Discussion}

The spatial distribution of repolarization changes induced by neural stimulation was determined by means of QRST isoarea maps obtained from multiple atrial recording sites, as done previously with respect to ventricular electrical events. ${ }^{28}$ The data so obtained indicate that the autonomic nervous system displays a heterogeneity of atrial electrical responses when its various extrinsic and intrinsic neural elements are stimulated electrically.

Consistent QRST area changes were induced in the right atrial free wall during stimulation of the right and left vagosympathetic complexes, as well as the right atrial ganglionated plexus. These changes were associated with a caudal shift of the atrial pacemaker site as seen in corresponding isochronal maps (see Fig. 2). Although parasympathetic stimulation induced such changes in the sequence of electrical activation of the atrium, the similarities between QRST area maps generated during spontaneous rhythms and those obtained during constant left atrial pacing suggest that the QRST area of local atrial electrograms is not affected by conduction changes. Similar vagal effects on atrial pacemakers have been reported previously, as well as the induction of atrial fibrillation by vagal stimulation., ${ }^{43-35}$ QRST area changes were also induced by vagal stimulation in other atrial regions (see Fig. 4). However, it is noteworthy that no preferential distribution of the right or left vagosympathetic complexes on either the right or left atrium was clearly demonstrated.

Our data also indicate that stimulation of loci in the right atrial ganglionated plexus induced changes in regions of the right atrium remote from the sinus node region and also in the left atrium (Fig. 4, Fig. $5, B, C$, and $D$, and Fig. 6), indicating that neural elements in the right atrial ganglionated plexus project to many more atrial sites than suggested previously. ${ }^{12,15,16,18,36,37}$ Efferent parasympathetic innervation of the canine sinoatrial node has been reported to arise from parasympathetic neurons in the right atrial ganglionated plexus. ${ }^{10-12,15-18,36}$ Furthermore, data reported herein demonstrate that 
(1) the right atrial ganglionated plexus contains not only efferent parasympathetic neural elements but sympathetic ones as well and (2) all of these neural elements when activated can modify tissues throughout both atria. These data are in accord with those describing atrial contractile responses to stimulation of the right atrial ganglionated plexus. ${ }^{14}$

Atrial effects elicited by stellate ganglion stimulation were of a lesser magnitude and occurred with a lower incidence. Although small, QRST area changes induced by stellate ganglion stimulation were regionally distributed (see Figs. 4 and 5 ). The fact that right stellate ganglion stimulation affected the sinus rate in all preparations whereas left stellate ganglion stimulation produced such chronotropic responses in only 11 of the 16 preparations is consistent with the classic notion of preferential innervation of the sinus node from the right stellate ganglion. ${ }^{6,38}$ This was corroborated by the fact that, in a preparation in which left stellate ganglion stimulation did not affect sinus rate, the repolarization changes were restricted to the left atrium and did not extend to the sinus node region.

QRST area changes identified in the present study are consistent with heterogeneous modifications of repolarization. The nonuniform distribution of atrial refractory period shortening is a widely known effect of vagal stimulation. ${ }^{19-21}$ Using refractory period determinations by the extrastimulus technique, Zipes, Mihalik, and Robbins ${ }^{20}$ have shown that right vagal stimulation elicits greater effects in the right atrium than the left atrium and that shortening of atrial refractory periods are more pronounced during right than left vagal stimulation. The present study not only supported these findings, but also provided information depicting the complexity of atrial parasympathetic innervation.

QRST areas changes of opposite polarity were induced in different regions of the right atrium or between the right and left atria when efferent autonomic neural structures were activated (see Fig. 5). Because no detailed information is available concerning the anatomic patterns of sympathetic or parasympathetic nerve projections to the atrial subepicardial, subendocardial, or intramural regions, it is presumed that the direction of changes induced in any region, whether positive or negative, was indicative of local neural effects on repolarization regardless of the polarity of changes. Although dominant polarity changes did occur (see Fig. 4), positive or negative changes were elicited in any given region during stimulation of a specific neural element in different dogs (see Fig. 5). The medial right atrial wall (region 1, which contains the sinoatrial node) was the only atrial region in which changes of a given polarity (positive) consistently occurred when similar neural elements were stimulated. Many of the neural structures stimulated contain mixed sympathetic and parasympathetic neural elements (vagosympathetic complexes and right atrial ganglionated plexus). Similarity of results elicited in the cranial left atrium when the right atrial ganglionated plexus or the right stellate ganglion was stimulated could be interpreted as implying that sympathetic efferent neural elements arising from the stellate ganglion course through the right atrial ganglionated plexus.

Surgical implications. The efferent neuronal projections to the atria were defined with a high degree of spatial and temporal resolution by mapping atrial QRST area changes, revealing a highly individualized and consistent pattern of atrial innervation in each preparation. When several preparations were considered, marked interindividual variability was noted; however, common features were identified between preparations. The present study demonstrated a wider atrial distribution of individual right- or left-sided autonomic neurons than previously appreciated. This method may be helpful to investigate the effects of currently used surgical incisions on atrial innervation. For instance, the classic approach to the mitral valve made through the fat pad containing the right atrial ganglionated plexus is likely to alter parasympathetic innervation of the sinus node and right atrial free wall. Furthermore, the data demonstrating considerable overlap of postganglionic neural elements suggest that the feasibility of selective denervation of specific portions of the atrium is uncertain. Extension of such structure-function studies to patients may expand the surgeon's understanding of mechanisms involved in disturbances of cardiac rhythm or dynamics occurring as either primary events or postoperative complications.

\section{REFERENCES}

1. Murphy DA, Armour JA. Human cardiac nerve stimulation. Ann Thorac Surg 1992;54:502-6.

2. Carlson MD, Geha AS, Hsu J, et al. Selective stimulation of parasympathetic nerve fibers to the human sinoatrial node. Circulation 1992;85:1311-7.

3. Ali IM, Butler CK, Armour JA, Murphy DA. Modification of supraventricular tachyarrhythmias by stimulating atrial neurons. Ann Thorac Surg 1990;50:251-6. 
4. Pagé PL, Hassanalizadeh $H$, Cardinal $R$. Transitions among atrial fibrillation, atrial flutter, and sinus rhythm during procainamide infusion and vagal stimulation in dogs with sterile pericarditis. Can J Physiol Pharmacol 1991;69:15-24.

5. Niemela MJ, Airaksinen KE, Tahvanainen KU, Linnaluoto MK, Takkunen JT. Effect of coronary artery bypass grafting on cardiac parasympathetic nervous function. Eur Heart J 1992;13:932-5.

6. Randall WC, Priola DV, Ulmer RHG. A functional study of the distribution of cardiac sympathetic nerves. Am J Physiol 1963;205:1227-31.

7. Seis WP, Kaye MP, Randall WC. Major autonomic pathways to the atria and S-A and A-V nodes of the canine heart. Am J Physiol 1973;224:202-8.

8. Stuesse SL, Wallick DW, Levy MN. Autonomic control of right atrial contractile strength in the dog. Am J Physiol 1979;236:H860-5.

9. Schuessler RB, Boineau JP, Wylds AC, Hill DA, Miller CB, Roeske WR. Effect of canine cardiac nerves on heart rate, rhythm and pacemaker location. Am J Physiol 1986;250:H630-44.

10. Lazzara R, Scherlag BJ, Robinson MJ, Samet P. Selective in situ parasympathetic control of the canine sinoatrial and atrioventricular nodes. Circ Res 1973; 32:393-401.

11. Randall WC, Ardell JL, Calderwood D, Milosavljevic M, Goyal SC. Parasympathetic ganglia innervating the canine atrioventricular nodal region. J Autonom Nerv Syst 1986;16:311-23.

12. Randall WC, Ardell JL, Wurster RD, Miloslavljevic M. Vagal postganglionic innervation of the canine sinoatrial node. J Auton Nerv Syst 1987;20:13-23.

13. Gagliardi M, Randall WC, Bieger D, Wurster RD, Hopkins DA, Armour JA. Activity of in vivo canine cardiac plexus neurons. Am J Physiol 1988;255:H789800.

14. Butler CK, Smith FM, Cardinal R, Murphy DA, Hopkins DA, Armour JA. Cardiac responses to electrical stimulation of discrete loci in canine atrial and ventricular ganglionated plexi. Am J Physiol 1990;259: H1365-73.

15. Ardell JL, Randall WC. Selective vagal innervation of sinoatrial and atrioventricular nodes in canine hearts. Am J Physiol 1986;251:H764-73.

16. Randall WC, Ardell JL. Selective parasympathectomy of automatic and conductile tissues of the canine heart. Am J Physiol 1985;248:H61-8.

17. Randall WC, Wurster RD, Duff M, O'Toole MF, Wehrmacher W. Surgical interruption of postganglionic innervation of the sinoatrial nodal region. $J$ Thorac Cardiovasc Surg 1991;101:66-74.

18. Mick JD, Wurster RD, Duff M, Weber M, Randall WC, Randall DC. Epicardial sites for vagal mediation of sinoatrial function. Am $J$ Physiol 1992;262: H1401-6.
19. Alessi R, Nusynowitz M, Abildskov JA, Moe GK. Nonuniform distribution of vagal effects on the atrial refractory period. Am J Physiol 1958;194:406-10.

20. Zipes DP, Mihalik MJ, Robbins GT. Effects of selective vagal and stellate ganglion stimulation on atrial refractoriness. Cardiovasc Res 1974;8:647-55.

21. Takei M, Furukawa Y, Narita M, et al. Synergistic nonuniform shortening of atrial refractory period induced by autonomic stimulation. Am J Physiol 1991;261:H1988-93.

22. Abildskov JA, Evans AK, Lux RL, Burgess MJ. Ventricular recovery properties and the QRST deflection area in cardiac electrograms. Am J Physiol 1980;239:H227-31.

23. Millar CK, Kralios FA, Lux, RL. Correlation between refractory periods and activation-recovery intervals from electrograms: effects of rate and adrenergic interventions. Circulation 1985;72:1372-9.

24. Russell DC, Dart A. T wave amplitude as a quantitative index of regional myocardial sympathetic responsiveness. J Cardiovasc Pharmacol 1986;8:629-35.

25. Cardinal R, Savard P, Armour JA, et al. Ventricular patterns of efferent sympathetic innervation and origins of sympathetically induced ventricular tachycardias. In: Shenasa M, Borggrefe M, Breithardt G, eds. Cardiac mapping. Mt. Kisco, N.Y.: Futura, 1993:26373.

26. Wilson FN, Macleod AG, Barker PS, Johnston DF. The determination and the significance of the areas of the ventricular deflections of the electrocardiogram. Am Heart J 1934;10:46-61.

27. Hanashima K, Kubota I, Ozawa T, et al. Effect of altered activation sequence on epicardial QRST area and refractory period in dogs. Circulation 1991;84: 1346-53.

28. Savard P, Cardinal R, Nadeau RA, Armour JA. Epicardial distribution of ST segment and $T$ wave changes produced by stimulation of intrathoracic ganglia or cardiopulmonary nerves in dogs. J Auton Nerv Syst 1991;34:47-58.

29. Bonneau G, Tremblay G, Savard P, et al. An integrated system for intraoperative cardiac activation mapping. IEEE Trans Biomed Eng 1987;BME-34: 415-23.

30. Wilkinson L. SYGRAPH: The System for Graphics. Evanston, Ill.: SYSTAT, Inc., 1990:172-87.

31. Olfert ED, Cross BM, McWilliams DVM. Guide to the care and use of experimental animals, 2nd ed. Ottawa: Canadian Council on Animal Care, 1993.

32. Hoffman BF, Cranefield PF. The electrophysiology of the heart. New York: McGraw-Hill, 1960:42-74.

33. Meek WJ, Eyster JAE. Experiments on the origin and propagation of the impulse in the heart. IV. The effect of vagal stimulation and of cooling on the location of the pacemaker within the sinoauricular node. Am J Physiol 1914;34:368-83. 
34. Watanabe H, Perry JB, Pagé P, Savard P, Nadeau R. Vagal effects on sinoatrial and atrial conduction studied with epicardial mapping in dogs: the influence of pacemaker shifts on the measurement of sinoatrial conduction time. Can' J Physiol Pharmacol 1985;63: 113-21.

35. Boineau JP, Miller CB, Schuessler RB, et al. Activation sequence and potential distribution maps demonstrating multicentric atrial impulse origin in dogs. Circ Res 1984;54:332-47.

36. Furakawa Y, Narita M, Takei M, Kobayashi O,
Haniuda M, Chiba S. Differential intracardiac sympathetic and parasympathetic innervation to the SA and AV nodes in anesthetized dog hearts. Jpn J Pharmacol 1991;55:381-90.

37. Wallick DW, Martin PJ. Separate parasympathetic control of the heart rate and atrioventricular conduction of dogs. Am J Physiol 1990;259:H536-42.

38. Schwartz PJ. Sympathetic imbalance and cardiac arrhythmias. In: Randall WC, ed. Nervous control of cardiovascular function. New York: Oxford University Press, 1993:225-52. 